\title{
The impact of acute air pollution fluctuations on bronchiectasis pulmonary exacerbation: a case-crossover analysis
}

\author{
Pieter C. Goeminne ${ }^{1,2}$, Bianca Cox ${ }^{3}$, Simon Finch ${ }^{4}$, Michael R. Loebinger ${ }^{5}$, \\ Pallavi Bedi ${ }^{6}$, Adam T. Hill 6,7 , Tom C. Fardon ${ }^{4}$, Kees de Hoogh ${ }^{8,9}$, \\ Tim S. Nawrot ${ }^{3,10}$ and James D. Chalmers ${ }^{4}$
}

\begin{abstract}
Affiliations: ${ }^{1}$ Dept of Respiratory Medicine, AZ Nikolaas, Sint-Niklaas, Belgium. ${ }^{2}$ Dept of Respiratory Medicine, UZ Leuven, Leuven, Belgium. ${ }^{3}$ Centre for Environmental Sciences, Hasselt University, Diepenbeek, Belgium. ${ }^{4}$ Scottish Centre for Respiratory Research, University of Dundee, Dundee, UK. ${ }^{5}$ Royal Brompton Hospital and The National Heart and Lung Institute, Imperial College London, London, UK. 'University of Edinburgh/MRC Centre for Inflammation Research, Queen's Medical Research Institute, Edinburgh, UK. ${ }^{7}$ Dept of Respiratory Medicine, Royal Infirmary of Edinburgh, Edinburgh, UK. ${ }^{8}$ Swiss Tropical and Public Health Institute, Basel, Switzerland. ${ }^{9}$ University of Basel, Basel, Switzerland. ${ }^{10}$ Dept of Public Health and Primary Care, Leuven University, Leuven, Belgium.
\end{abstract}

Correspondence: Pieter C. Goeminne, Moerlandstraat 1, B-9100, St-Niklaas, Belgium. E-mail: pieter. goeminnedaznikolaas.be

@ERSpublications

Acute air pollution fluctuations are associated with increased exacerbation risk in patients with bronchiectasis http://ow.ly/ehiN30khUOJ

Cite this article as: Goeminne PC, Cox B, Finch S, et al. The impact of acute air pollution fluctuations on bronchiectasis pulmonary exacerbation: a case-crossover analysis. Eur Respir J 2018; 52 : 1702557 [https:// doi.org/10.1183/13993003.02557-2017].

ABSTRACT In bronchiectasis, exacerbations are believed to be triggered by infectious agents, but often no pathogen can be identified. We hypothesised that acute air pollution exposure may be associated with bronchiectasis exacerbations.

We combined a case-crossover design with distributed lag models in an observational record linkage study. Patients were recruited from a specialist bronchiectasis clinic at Ninewells Hospital, Dundee, UK.

We recruited 432 patients with clinically confirmed bronchiectasis, as diagnosed by high-resolution computed tomography. After excluding days with missing air pollution data, the final model for particles with a $50 \%$ cut-off aerodynamic diameter of $10 \mu \mathrm{m}$ (PM10) was based on 6741 exacerbations from 430 patients and for nitrogen dioxide $\left(\mathrm{NO}_{2}\right)$ it included 6248 exacerbations from 426 patients. For each $10 \mu \mathrm{g} \cdot \mathrm{m}^{-3}$ increase in $\mathrm{PM} 10$ and $\mathrm{NO}_{2}$, the risk of having an exacerbation that same day increased significantly by $4.5 \%$ (95\% CI $0.9-8.3$ ) and 3.2\% (95\% CI $0.7-5.8$ ) respectively. The overall (lag zero to four) increase in risk of exacerbation for a $10 \mu \mathrm{g} \cdot \mathrm{m}^{-3}$ increase in air pollutant concentration was $11.2 \%$ (95\% CI 6.0-16.8) for PM10 and 4.7\% (95\% CI 0.1-9.5) for $\mathrm{NO}_{2}$. Subanalysis showed higher relative risks during spring (PM10 1.198 (95\% CI 1.102-1.303), $\mathrm{NO}_{2} 1.146$ (95\% CI 1.035-1.268)) and summer (PM10 2.142 (95\% CI 1.785-2.570), $\mathrm{NO}_{2} 1.352$ (95\% CI 1.140-1.602)) when outdoor air pollution exposure would be expected to be highest.

In conclusion, acute air pollution fluctuations are associated with increased exacerbation risk in bronchiectasis. 


\section{Introduction}

Exacerbations are key events in the natural history of bronchiectasis and are associated with an increased risk of mortality, hospital admissions, lung function decline and death [1,2]. The causes of bronchiectasis exacerbations are not known, although they are presumed to be primarily bacterial or viral and guidelines recommend that all exacerbations are treated with antibiotics [3]. Therapies aiming to reduce exacerbation frequency in bronchiectasis, however, have generally had modest effects and there is a high burden of exacerbation despite treatment. European registry data shows a median of two exacerbations per patient per year [4]. It is therefore important to gain further understanding of the causes of bronchiectasis exacerbations. Research shows that exposure to air pollution causes an inflammatory response. One study by MCCREANOR et al. [5] showed that patients with asthma walking for $2 \mathrm{~h}$ along a busy London street not only showed higher pollution exposure than their controls who walked through a nearby park, but they had decreased forced expiratory volume in $1 \mathrm{~s}$ (FEV1) and forced vital capacity (FVC). These changes were accompanied by a significant increase in neutrophilic inflammation measured by sputum myeloperoxidase, also known to be a key mediator in bronchiectasis [6].

Acute and chronic air pollution exposures have both been linked with pulmonary exacerbations in patients suffering from cystic fibrosis (CF). Acute fluctuations play a role in triggering pulmonary exacerbations in CF $[7,8]$. Furthermore, proximity to major roads predicts exacerbations and annual average exposure to air pollution is associated with an increased risk of a pulmonary exacerbation and a decline in lung function $[9,10]$.

For non-CF bronchiectasis, from now on referred to as bronchiectasis, data are scant. We recently showed that residential proximity to a major road and distance-weighted traffic density within 100 and $200 \mathrm{~m}$ was associated with the risk of mortality [11]. To our knowledge, there are no data on the effect of acute air pollution on bronchiectasis pulmonary exacerbations.

This study aimed to determine the impact of acute air pollution fluctuations on the risk of pulmonary exacerbation in patients with bronchiectasis.

\section{Materials and methods}

\section{Patient population and patient data linkage}

This article describes a case-crossover observational record linkage study of patients with clinically confirmed bronchiectasis, as diagnosed by high-resolution computed tomography (HRCT), who attended a regional specialist clinic for bronchiectasis patients at Ninewells Hospital, Dundee, UK. The hospital is a national reference centre and therefore patients came from other cities around the country including Aberdeen, Dundee, Kirkcaldy, Perth and Falkirk. The study was approved by the local research ethics committee (study number: 14/SS/1101) and all patients gave written informed consent to participate. Unique personal identifier codes were used to extract electronic medical records from an administrative database that covers more than 800000 patients (approximately 16\% of the Scottish population) from the date of first diagnosis of bronchiectasis (defined as the date of the initial diagnostic HRCT scan). Linkage included data from January 01, 2000 to October 31, 2014 including all community drug prescriptions, hospital stays, diagnoses, interventions, laboratory tests (including sputum microbiology), radiology and deaths. Patients were also included in the Tayside Respiratory Disease Information System (TARDIS) which provides annual data on spirometry, microbiology, respiratory symptoms and respiratory treatments, and has been used for multiple previous record linkage studies [12-14].

\section{Exacerbation definition}

During the study period there was no agreed definition of exacerbation of bronchiectasis, but most operational definitions previously required the acute prescription of antibiotics [1]. During the study period, exacerbations were identified as an acute prescription of antibiotics for episodes coded as exacerbation of bronchiectasis or lower respiratory tract infection at the time of clinical presentation. This method of identification of exacerbations was prospectively validated against exacerbations treated in a prospective cohort and was 100\% sensitive and $87 \%$ specific (more details are provided in the supplementary material). Severe exacerbations were defined as admissions to hospital for exacerbation of bronchiectasis. The first day of antibiotic prescription was considered to be the start date of the exacerbation.

\section{Exposure data}

Daily concentrations of particles with a $50 \%$ cut-off aerodynamic diameter of $10 \mu \mathrm{m}$ (PM10) and nitrogen dioxide $\left(\mathrm{NO}_{2}\right)$ used for the analysis were measured during the study period at the Aberdeen urban background site, which is part of the UK's Automatic Urban and Rural Network (AURN). Days with missing air pollution data were excluded and, as climate is a known confounder of the association between 
air pollution and respiratory diseases [15], data on mean air temperature and relative humidity were obtained from the UK Meteorological Office. For temperature, we used data from the measuring stations Dyce, Mylnefield, Kinross, Dalwhinnie and Tyndrum for the postcode areas Aberdeen, Dundee, Kirkcaldy, Perth and Falkirk, respectively. As humidity data are not available for Mylnefield and Kinross, humidity recorded at the stations of Strathallan Airfield and Leuchars were used for the postcode areas Dundee and Kirkcaldy, respectively.

\section{Statistical analysis}

The case-crossover design is widely used for analysing short-term exposures with acute outcomes [16]. It is a variant of the matched case-control study, where each subject serves as its own control so that known and unknown time-invariant confounders are inherently adjusted for by study design [17]. This design samples only cases (exacerbations of bronchiectasis in this study) and compares each subject's exposure in a time period just before a case event (the hazard period) with that subject's exposure at other times (the control periods). Selection bias is avoided by applying a bidirectional time-stratified design [18]. Control days are taken from the same calendar month and year as the case day (the day of the bronchiectasis exacerbation), both before and after the case, thus controlling for long-term trends and season by design. Cases and controls were additionally matched by day of the week to control for any weekly patterns in air pollution or bronchiectasis. In the main analysis recurrent event data were pooled, considering exacerbations as the unit of analysis rather than persons and assuming that within-subject correlation is completely accounted for by subject-specific variables (observed or unobserved) [19].

To investigate the association between bronchiectasis and air pollution exposure up to 4 days before the exacerbation (lag zero to four), we combined the case-crossover design with distributed lag models, using separate models for PM10 and $\mathrm{NO}_{2}$. A distributed lag (non-linear) model is defined through a "cross-basis function", which allows the simultaneous estimation of a (non-linear) exposure-response association as well as non-linear effects across lags, the latter being termed a "lag-response association". This study applies recent extensions of the distributed lag model methodology beyond aggregated time series data [20], specifically implementing them in a conditional logistic regression model with individual-level exposure measures (at least for temperature). We assumed a linear association between air pollution exposure and bronchiectasis and the lag structure was modelled with a natural cubic spline with three degrees of freedom (df). The knots in the lag space were set at equally spaced values in the log scale of lags to allow more flexible lag effects at shorter delays [21].

We also included a cross-basis for mean temperature in the model to capture the (potentially delayed) effects of heat and cold on bronchiectasis exacerbations. The maximum lag was set to 25 days. We used a natural cubic spline with five df for the temperature-exacerbation function and a natural cubic spline with six df (with knots at equally spaced values in the log scale) for the lag structure. Spline knots for temperature were placed at equally spaced values of the actual temperature range to allow enough flexibility in the two ends of the temperature distribution.

In a secondary analysis we studied the effects stratified by Pseudomonas aeruginosa infection status (yes or no), by bronchiectasis severity index (BSI; mild: $\leqslant 4$, moderate: $5-8$, severe: $\geqslant 9$ ) and by deprivation index (divided into quintiles from the Scottish Index of Multiple Deprivation, a score from one to five where a lower index indicates higher deprivation), hospitalisation (yes or no), chronic macrolide use at the time of exacerbation (yes or no) and season (spring: March-May; summer: June-August; autumn: September-November; winter: December-February) [1, 22, 23]. In sensitivity analyses we increased the maximum lag to six. We also used an unconstrained distributed lag model, that is, a model in which each lag is entered as a separate variable [24]. Due to the correlation between air pollution concentrations on days close together, the unconstrained distributed lag model will result in unstable estimates for the individual lags; however, it is known as being more flexible and less prone to bias for the estimate of the overall effect [24]. We also explored potential confounding by temperature by excluding the temperature cross-basis from the model and explored potential confounding by humidity up to 4 days before the exacerbation by adding a cross-basis with a linear humidity-bronchiectasis function and a natural cubic spline with three $\mathrm{df}$ to model the lag structure. In a last sensitivity analysis, we used only one exacerbation per person (the first), such that no assumption on the correlation among multiple events was needed .

We calculated relative risks (RRs) of bronchiectasis exacerbation for a $10 \mu \mathrm{g} \cdot \mathrm{m}^{-3}$ increase in air pollutant concentrations. In our analysis, this accords to almost one SD. Reported estimates, computed as the overall cumulative risk accounting for the zero to four lag period, are presented as percentage change in bronchiectasis with corresponding 95\% CIs. All analyses were performed with the R statistical software package "dlnm" (The R Foundation for Statistical Computing, Vienna, Austria) [20]. 


\section{Results}

Patient population

Between January 06, 2000 and October 02, 2014, 432 patients had a total of 7777 exacerbations. After excluding days with missing air pollution data, the final model for PM10 was based on 6741 exacerbations from 430 patients and the final model for $\mathrm{NO}_{2}$ included 6248 exacerbations from 426 patients. The population was predominantly female (60.2\%) with a median age of 68 years (IQR 59-74). Chronic $P$. aeruginosa infection during the follow-up period was seen in $13.9 \%$ of the patient population. The BSI had an equal distribution of mild, moderate and severe risk patients (mild: $28.1 \%$; moderate: $39.5 \%$; severe: $32.3 \%$ ) (table 1 ).

\section{Ambient air pollution}

The descriptive statistics for the overall daily number of exacerbations, mean temperature and ambient air pollutants are shown in table 2. Mean temperature ranged from -11.5 to $21.3{ }^{\circ} \mathrm{C}$, with an average of $8.1{ }^{\circ} \mathrm{C}$. Average (range) PM10 and $\mathrm{NO}_{2}$ concentrations were $15.8 \mu \mathrm{g} \cdot \mathrm{m}^{-3}(1.0-82.6)$ and $25.5 \mu \mathrm{g} \cdot \mathrm{m}^{-3}(1.7-85.7)$, respectively. To highlight sufficient variation around a non-zero mean value, as suggested in case-crossover studies [25], table 2 also presents the "relevant exposure term", which is the absolute difference between each pollutant's level on the case day and its average concentration over the control days. The Spearman correlation coefficient between $\mathrm{PM} 10$ and $\mathrm{NO}_{2}$ was 0.35 . The correlation with mean temperature was 0.05 for $\mathrm{PM} 10$ and -0.30 for $\mathrm{NO}_{2}$.

\section{Effects of air pollution on exacerbation risk}

The three-dimensional plots show the association between bronchiectasis and PM10 and $\mathrm{NO}_{2}$ concentrations over the lag days (figure 1). The effect of air pollution on bronchiectasis exacerbations was found to be acute, with the highest RRs on the day of exposure (lag zero). Figure 2 presents the lag-specific RR for bronchiectasis associated with a $10 \mu \mathrm{g} \cdot \mathrm{m}^{-3}$ increase in air pollutant concentrations. Significant RRs were observed at lag zero and lag one for PM10, and at lag zero for $\mathrm{NO}_{2}$. The risk of having an exacerbation increased by $4.5 \%$ (95\% CI $0.9-8.3)$ and by $3.2 \%$ (95\% CI $0.7-5.8$ ) for each $10 \mu \mathrm{g} \cdot \mathrm{m}^{-3}$ increase in same-day (lag zero) $\mathrm{PM}_{10}$ and $\mathrm{NO}_{2}$, respectively. Cumulative effects of air pollutant concentrations on bronchiectasis are presented in table 3. The overall (lag zero to four) increase in the risk

\section{TABLE 1 Patient characteristics of the studied population}

\begin{tabular}{|c|c|}
\hline Characteristic & Result \\
\hline Patients & 430 \\
\hline Female sex & $259(60.2)$ \\
\hline Age years & $68(59-74)$ \\
\hline Exacerbations & 7319 \\
\hline \multicolumn{2}{|c|}{ Average exacerbations per patient } \\
\hline Over the study period & $13.5(7-25)$ \\
\hline Per year & $0.91(0.47-1.68)$ \\
\hline FEV $1 \%(n=429)$ & $71(50-90)$ \\
\hline \multicolumn{2}{|l|}{ Deprivation index $(n=419)^{\#}$} \\
\hline Index 1 & $86(20.5)$ \\
\hline Index 2 & 70 (16.7) \\
\hline Index 3 & $73(17.4)$ \\
\hline Index 4 & $115(27.5)$ \\
\hline Index 5 & 75 (17.9) \\
\hline \multicolumn{2}{|l|}{ Bronchiectasis severity index } \\
\hline Mild $(\leqslant 4)$ & $121(28.1)$ \\
\hline Moderate (5-8) & 170 (39.5) \\
\hline Severe $(\geqslant 9)$ & 139 (32.3) \\
\hline Median & $6(4-10)$ \\
\hline \multicolumn{2}{|c|}{ Chronic bacterial infection at enrolment } \\
\hline Haemophilus influenzae & $136(31.6)$ \\
\hline Staphylococcus aureus & $34(7.9)$ \\
\hline Moraxella catarrhalis & 53 (12.3) \\
\hline Enterobacteriaceae & 59 (13.7) \\
\hline Pseudomonas aeruginosa & 60 (13.9) \\
\hline
\end{tabular}

Data are presented as $\mathrm{n}, \mathrm{n}(\%)$, or median (IQR). FEV1: forced expiratory volume in $1 \mathrm{~s}$; IQR: interquartile range. ${ }^{\#}$ : a lower index value indicates higher deprivation. 
TABLE 2 Summary statistics for daily bronchiectasis exacerbations, mean temperature and air pollution levels, and for the absolute differences between the daily levels of each variable (case days) and the average levels over control days

\begin{tabular}{|c|c|c|c|c|}
\hline Variable & Mean & SD & Minimum & Maximum \\
\hline Exacerbations per day ${ }^{\#}$ & 3.1 & 3.7 & 0.0 & 42.0 \\
\hline \multicolumn{5}{|l|}{ Exposure on case days } \\
\hline Temperature ${ }^{\# \circ} \mathrm{C}$ & 8.1 & 5.1 & -11.5 & 21.3 \\
\hline $\mathrm{PM} 10^{\#} \mu \mathrm{g} \cdot \mathrm{m}^{-3}$ & 15.8 & 9.4 & 1.0 & 82.6 \\
\hline $\mathrm{NO}_{2}{ }^{\Upsilon} \mu \mathrm{g} \cdot \mathrm{m}^{-3}$ & 25.5 & 13.6 & 1.7 & 85.7 \\
\hline \multicolumn{5}{|c|}{ Exposure difference between case days and average over control days ${ }^{+}$} \\
\hline Temperature ${ }^{\#}{ }^{\circ} \mathrm{C}$ & 10.2 & 8.9 & 0.0 & 53.7 \\
\hline $\mathrm{PM} 10^{\#} \mu \mathrm{g} \cdot \mathrm{m}^{-3}$ & 7.2 & 7.1 & 0.0 & 62.8 \\
\hline $\mathrm{NO}_{2}{ }^{\Uparrow} \mu \mathrm{g} \cdot \mathrm{m}^{-3}$ & 2.3 & 1.8 & 0.0 & 13.6 \\
\hline
\end{tabular}

PM10: particles with a $50 \%$ cut-off aerodynamic diameter of $10 \mu \mathrm{m} ; \mathrm{NO}_{2}$ : nitrogen dioxide. ${ }^{\#}$ : summary statistics for cases with PM10 data available (exacerbations: $n=6741$; patients: $n=430$ ); ${ }^{\text {l: }}$ summary statistics for cases with $\mathrm{NO}_{2}$ data available (exacerbations: $n=6248$; patients: $n=426$ ); ${ }^{+}$: the relevant exposure term in a case-crossover design [25].

of an exacerbation for a $10 \mu \mathrm{g} \cdot \mathrm{m}^{-3}$ increase in air pollutant concentration was $11.2 \%$ (95\% CI 6.0-16.8) for PM10 and 4.7\% (95\% CI 0.1-9.5) for $\mathrm{NO}_{2}$.

Stratification according to chronic $P$. aeruginosa infection gave almost identical estimates in both groups, but CIs for the infected group were wider. In chronically infected patients the RRs for bronchiectasis exacerbations associated with a $10 \mu \mathrm{g} \cdot \mathrm{m}^{-3}$ increase in pollutant concentration were 1.115 (95\% CI 0.989 1.257) for PM10 and 1.046 (95\% CI 0.937-1.169) for $\mathrm{NO}_{2}$. In P. aeruginosa naïve patients, the RRs for bronchiectasis exacerbations associated with a $10 \mu \mathrm{g} \cdot \mathrm{m}^{-3}$ increase in pollutant concentration were 1.114 (95\% CI 1.056-1.175) for PM10 and 1.046 (95\% CI 0.996-1.098) for $\mathrm{NO}_{2}$ (figure 3). Similarly, no difference was found between groups when stratifying according to BSI, deprivation index, hospitalisation and chronic macrolide use at time of exacerbation (figure 3). There was however a seasonal variation in the association between air pollution and bronchiectasis, with higher RRs seen during spring (PM10 1.198 (95\% CI 1.102-1.303); $\mathrm{NO}_{2} 1.146$ (95\% CI 1.035-1.268)) and especially during summer (PM10 2.142 (95\% CI 1.785-2.570); $\mathrm{NO}_{2} 1.352$ (95\% CI 1.140-1.602)) as compared to autumn (PM10 0.922 (95\% CI $0.828-$
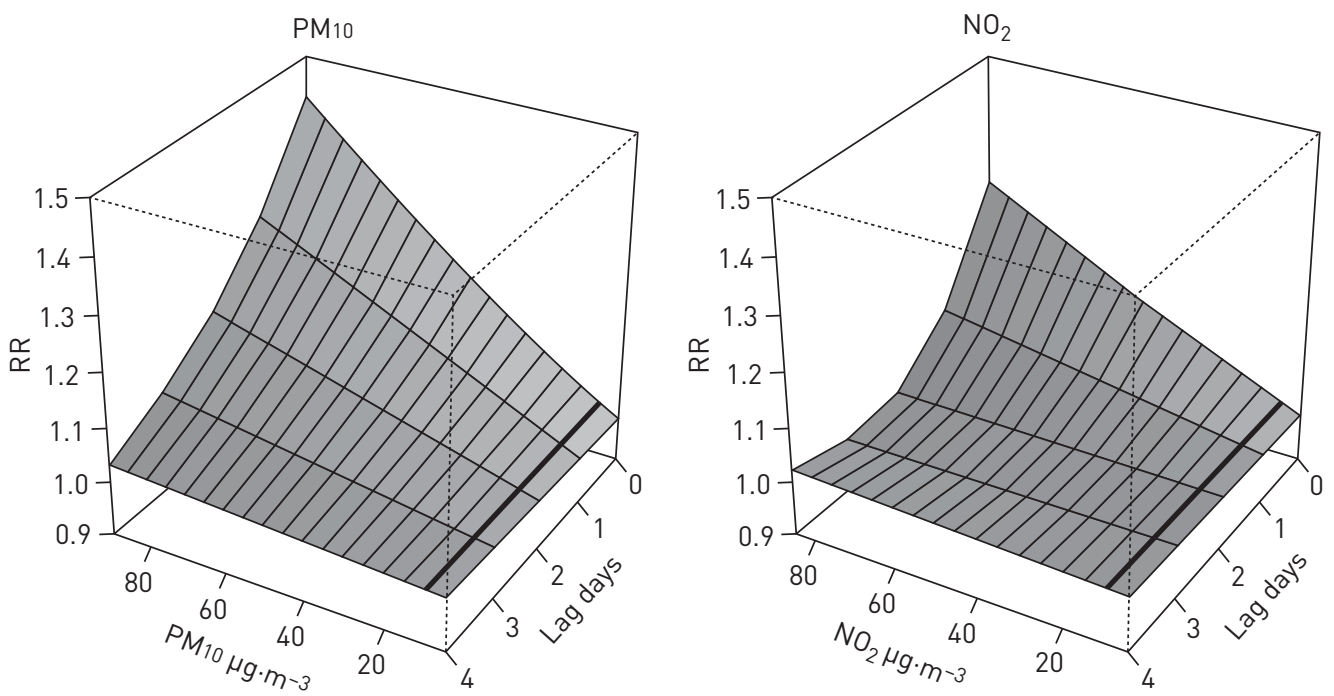

FIGURE 1 Exposure-lag-response surfaces for the association between bronchiectasis exacerbations and exposure to particles with a $50 \%$ cut-off aerodynamic diameter of $10 \mu \mathrm{m}$ (PM10) and nitrogen dioxide $\left(\mathrm{NO}_{2}\right)$. Relative risks (RRs) are relative to the reference value of $10 \mu \mathrm{g} \cdot \mathrm{m}^{-3}$ (bold line). A lag of zero is the day of exacerbation, while a lag of one is the day before the exacerbation. A lag of two, three and four corresponds to 2,3 and 4 days before the exacerbation. 

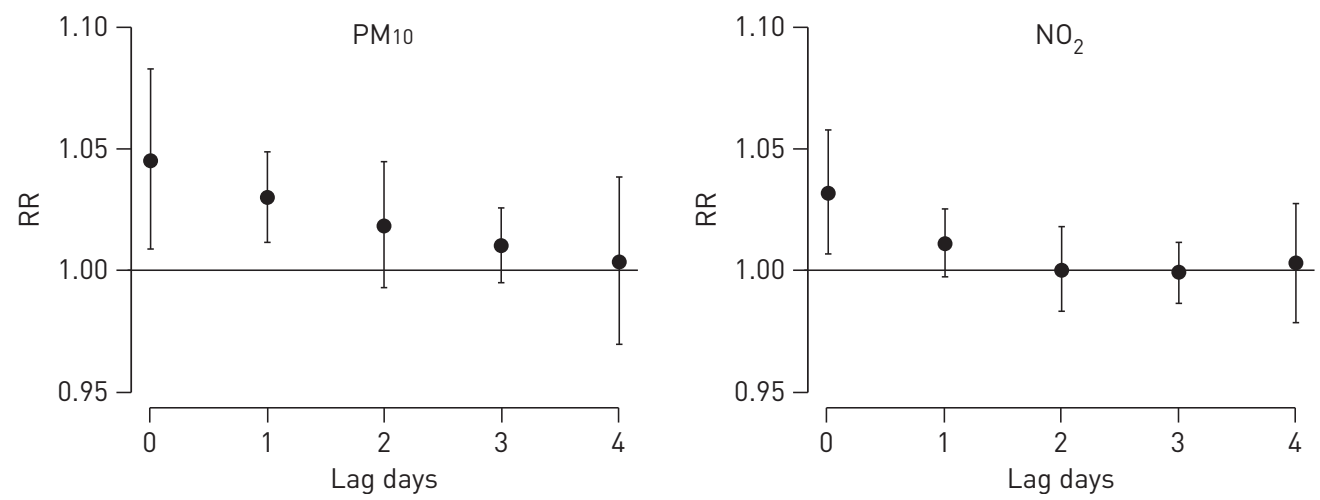

FIGURE 2 Lag-specific relative risks (RRs; 95\% Cl) for bronchiectasis exacerbations associated with a $10 \mu \mathrm{g} \cdot \mathrm{m}^{-3}$ increase in particles with a $50 \%$ cut-off aerodynamic diameter of $10 \mu \mathrm{m}$ (PM10) and nitrogen dioxide $\left(\mathrm{NO}_{2}\right)$.

1.027); $\mathrm{NO}_{2} 1.035$ (95\% CI 0.927-1.156)) and winter (PM10 0.984 (95\% CI 0.891-1.086); $\mathrm{NO}_{2} 0.941$ (95\% CI 0.880-1.005)) (figure 3).

Increasing the maximum lag to 6 days, using an unconstrained lag structure, excluding the cross-basis for temperature and including a cross-basis for humidity produced similar results for PM10, with cumulative lag zero to four effects ranging from $10.0 \%$ (95\% CI 5.1-15.1) to $11.8 \%$ (95\% CI 6.5-17.4) (table 4). Sensitivity analyses also showed robust results for $\mathrm{NO}_{2}$, except for a decrease in cumulative estimates when increasing the maximum lag to 6 days (3.9\% (95 CI -0.5 to 8.5 )) and when adding the humidity cross-basis (3.6\% (95\% CI -1.1 to 8.6$)$ ). Same-day (lag zero) estimates from these models, however, remained significant (3.5\% and $2.7 \%$, respectively). Limiting the analysis to the first exacerbation of each patient did not change the cumulative estimate for PM10 (11.1\% (95\% CI -9.2 to 36.0)) but resulted in an increase in the estimate for $\mathrm{NO}_{2}(9.0 \%(95 \% \mathrm{CI}-7.3$ to 28.1$))$ and a considerable loss in precision for both air pollutants.

\section{Discussion}

This is to our knowledge the first report on acute air pollution fluctuations and their effect on exacerbations in a population suffering from bronchiectasis. This case-crossover analysis shows that the risk of having an exacerbation increased significantly on days with higher air pollution. For each $10 \mu \mathrm{g} \cdot \mathrm{m}^{-3}$ increase in PM10 and $\mathrm{NO}_{2}$, the risk of having an exacerbation that same day increased significantly by $4.5 \%$ and $3.2 \%$, respectively. The overall increase in the risk of exacerbation for a $10 \mu \mathrm{g} \cdot \mathrm{m}^{-3}$ increase in air pollutant concentration was much higher, reaching $11.2 \%$ for $\mathrm{PM} 10$ and $4.7 \%$ for $\mathrm{NO}_{2}$.

The effects of air pollution fluctuations seem to be very acute and other researchers have found similar acute effects. TRAmuto et al. [26] investigated the effects of air pollution on emergency room visits for respiratory symptoms and found that $\mathrm{PM} 10$ and $\mathrm{NO}_{2}$, amongst other pollutants, were positively associated in a similar acute fashion. Further comparable acute effects of air pollution have been shown for other diseases as well. NAWROT et al. [16] determined that air pollution is an important trigger for acute myocardial infarction.

TABLE 3 Cumulative effects of particles with a $50 \%$ cut-off aerodynamic diameter of $10 \mu \mathrm{m}$ (PM10) and nitrogen dioxide $\left(\mathrm{NO}_{2}\right)$ on bronchiectasis exacerbations along the lag days. Estimates represent the percentage change $(95 \% \mathrm{Cl})$ in bronchiectasis exacerbations for a $10 \mathrm{\mu g} \cdot \mathrm{m}^{-3}$ increase in air pollutant concentration.

\begin{tabular}{lcc} 
Lag days & $\mathbf{P M 1 0}$ & $\mathbf{N O}_{\mathbf{2}}$ \\
\hline $\mathbf{0}$ & $4.5(0.9 ; 8.3)$ & $3.2(0.7 ; 5.8)$ \\
$\mathbf{0}-\mathbf{1}$ & $7.7(3.7 ; 11.8)$ & $4.4(1.3 ; 7.5)$ \\
$\mathbf{0}-\mathbf{2}$ & $9.7(5.4 ; 14.2)$ & $4.5(0.9 ; 8.1)$ \\
$\mathbf{0}-\mathbf{0}$ & $10.8(6.1 ; 15.7)$ & $4.4(0.4 ; 8.5)$ \\
$\mathbf{0}-\mathbf{4}$ & $11.2(6.0 ; 16.8)$ & $4.7(0.1 ; 9.5)$
\end{tabular}




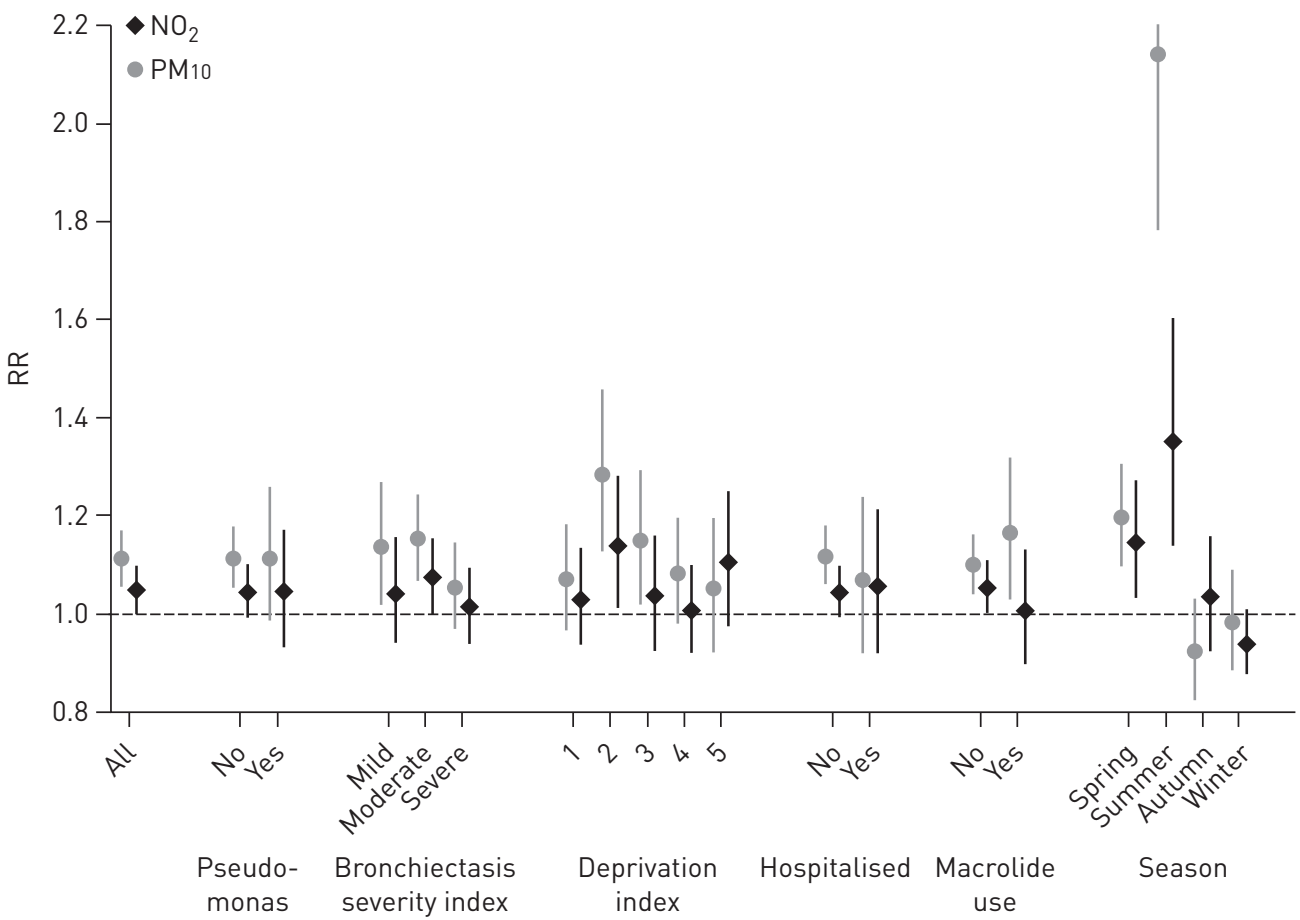

FIGURE 3 Cumulative relative risks (RRs; $95 \%$ CI) for bronchiectasis exacerbations associated with a $10 \mu \mathrm{g} \cdot \mathrm{m}^{-3}$ increase in particles with a $50 \%$ cut-off aerodynamic diameter of $10 \mu \mathrm{m}$ (PM10; grey circles) and nitrogen dioxide $\left(\mathrm{NO}_{2}\right.$; black diamonds) stratified according to Pseudomonas aeruginosa culture positivity, bronchiectasis severity index (BSI), deprivation index, hospitalisation, chronic macrolide use at time of exacerbation and season.

In our analysis, we could not show a predisposition towards the effects of air pollution fluctuation in any defined subgroup. Three subanalyses (chronic P. aeruginosa infection, deprivation index and BSI) showed overlapping CIs. These results seem to corroborate previous data from patients with CF, where $P$. aeruginosa status did not impact the effects of air pollution exposure [7]. Our data suggests that all subgroups of patients with bronchiectasis are potentially susceptible to the effects of acute air pollution.

Higher risks for bronchiectasis exacerbations associated with air pollution were, however, seen during spring and especially summer. We previously showed a similar stronger association between exacerbations and air pollution during the warmer months of the year in CF patients [7]. The reason remains unclear, but one interesting study showed that ambient pollution particles collected during the spring and the summer months were more potent at inducing inflammatory cytokines in isolated macrophages of rats

TABLE 4 Sensitivity analysis estimates for the cumulative lag zero to four effects of particles with a $50 \%$ cut-off aerodynamic diameter of $10 \mu \mathrm{m}$ (PM10) and nitrogen dioxide $\left(\mathrm{NO}_{2}\right)$ on bronchiectasis exacerbations. Estimates represent the percent change $(95 \% \mathrm{CI})$ in bronchiectasis exacerbations for a $10 \mu \mathrm{g} \cdot \mathrm{m}^{-3}$ increase in air pollutant concentration.

\begin{tabular}{lcc} 
Change in main model $^{\#}$ & PM10 & NO $_{\mathbf{2}}$ \\
\hline None & $11.2(6.0 ; 16.8)$ & $4.7(0.1 ; 9.5)$ \\
Maximum lag=6 days & $11.6(6.5 ; 16.9)$ & $3.9(-0.5 ; 8.5)$ \\
Unconstrained lag model & $11.8(6.5 ; 17.4)$ & $4.3(-0.2 ; 9.1)$ \\
No temperature correction & $10.0(5.1 ; 15.1)$ & $5.0(0.7 ; 9.5)$ \\
Humidity correction & $11.3(5.8 ; 17.2)$ & $3.6(-1.1 ; 8.6)$ \\
First event only & $11.1(-9.2 ; 36.0)$ & $9.0(-7.3 ; 28.1)$
\end{tabular}

\#: in the main analysis recurrent event data were pooled, while the distributed lag model had a maximum lag of 4 days, three degrees of freedom (df) for the lag-response function and was adjusted for mean temperature (cross-basis function with a maximum lag of 25 days, five $\mathrm{df}$ for the temperature-response function and six df for the lag-response function). 
compared with samples collected during the winter months [27]. Moreover, Scotland has a relatively cold climate meaning patients spend more time indoors during the autumn and winter months and our results may indicate that the effects are being driven by outdoor air pollution, such as traffic pollution exposure, during months that patients spend more time outdoors [28]. Finally, ambient temperature is associated with the prevalence of $P$. aeruginosa and lower lung function in patients with CF [29]. We speculate that during the colder months exacerbations are more likely viral in origin and less impacted by air pollution and that, during the warmer months, bacterial exacerbations are possibly worsened by air pollution or air pollution can have a direct effect on causing exacerbations because there are less viral infections during that period.

Our study has some limitations. One limitation is the lack of data on symptoms and days of symptoms before start of antibiotics, as well as the definition we used for exacerbation. We deemed the need for antibiotic treatment essential, thereby following initial definitions used in large double-blind, placebo-controlled trials in bronchiectasis [30,31]. However, an expert consensus definition was recently produced to assist further research. In this new definition the patient needs deteriorating symptoms and a decision to make a change in the patients' bronchiectasis treatment [32]. This suggests that non-antibiotic treatment started due to deteriorating symptoms might also fit the definition of a bronchiectasis exacerbation. This might have resulted in an underreporting of the number of exacerbations in our studied population.

Another limitation is the lack of data on particles with a 50\% cut-off aerodynamic diameter of $2.5 \mu \mathrm{m}$ (PM2.5) or black carbon, as these were not available for the region in enough monitoring stations for that time period. PM10 consists of PM2.5 and larger particles which are mainly of biological and crustal origin. These particles are capable of penetrating into the lower respiratory tract. Research has shown that the smaller PM2.5 fraction is more potent in causing respiratory effects [33]. However, high correlations between PM10 and PM2.5 in the region have been observed. For example, Auchencorth Moss pollution data between June 01, 2012 and December 31, 2015 show a PM10 and PM2.5 correlation coefficient of 0.86. Moreover, we added $\mathrm{NO}_{2}$ as black carbon is the particulate matter fraction that correlates best with $\mathrm{NO}_{2}$ [34]. $\mathrm{NO}_{2}$ is mainly emitted by combustion processes (e.g. vehicle engines, heating and power generation) and is therefore a good proxy for the mixture of traffic related air pollution.

A third limitation is the use of outdoor measurements to reflect a patient's personal exposure. It is possible that patients will spend more time indoors when media alert for high pollution concentrations. We do not believe this will have impacted our study in a significant way as media only alert peak pollution concentrations and we studied continuous exposure effects. Research has also shown a good correlation between outdoor and indoor variation. Studies show a very good correlation among the day-to-day changes in measurement stations and personal exposure $[35,36]$. We also know from previous data that spatial variability in PM10 is less important than temporal variability, as the latter is largely caused by weather changes [37]. However, added personal exposure based on commute and travelling was not evaluated due to ethical and privacy reasons and could therefore influence the results.

Ideally, to capture spatial and temporal variations we would have used air pollution monitoring sites which were located near where the participants live, were located away from local sources (so-called background sites) and were operated during the whole time period. During the time period concerned, the only site in Scotland fulfilling these requirements was an urban background site in Aberdeen. Therefore, all participants were assigned daily exposures from the same measurement site, giving us the temporal signal only. This could be a limitation, however, we don't believe that this influenced the results because evidence shows that temporal differences are much more determining than spatial differences in air pollution [37].

The four major strengths of this analysis are the large number of patients, the large number of exacerbation events due to the long-term follow-up, the case-crossover design and the use of distributed lag models. The case-crossover design is widely used for analysing short-term pollution exposure with acute outcomes. A case-crossover analysis is a variant of the case-control in which each subject serves as her or his own control [17]. This reduces the influence of confounding covariates. The use of distributed lag models is an important strength as their bi-dimensional structure simultaneously describes the association along the space of the exposure and in the additional dimension of the lags. This enables the investigation of the temporal pattern of the association in one single model and provides an estimate of the "overall" effect of the exposure incorporating delayed effects.

In our analysis we added deprivation index as a measure for socio-economic status as previous research in bronchiectasis has suggested that socio-economic status is linked with mortality and exacerbations [38]. We found no evidence that the effect of acute air pollution fluctuations on bronchiectasis pulmonary exacerbations was modified by deprivation index. To tackle the possible confounding effect of asthma or 
chronic obstructive pulmonary disease (COPD), we used a case-crossover analysis, as we did not expect that the chronic conditions of a person would change within the month of exacerbation or any other factor that slowly changes over time. In bronchiectasis, asthma and COPD are important comorbid conditions. The existence of asthma in patients with bronchiectasis is associated with an independent increase in the risk of bronchiectasis exacerbation and the relationship between asthma and pollution has been widely studied [39, 40]. Similarly, bronchiectasis with associated COPD not only shows increased mortality compared to bronchiectasis patients without COPD, but bronchiectasis patients with COPD also have higher rates of respiratory infection and hospitalisation [41, 42]. Additionally, air pollution increases hospitalisations and mortality in COPD [43].

This data adds to an increasing body of work suggesting that air pollution increases exacerbation risk in people with a respiratory illness. This presents a challenge for policymakers to address this growing problem. Pollution impacts the health of the whole population and certainly patients with a respiratory condition. Further action is needed as European data indicate that current exposure to particulate matter from anthropogenic sources leads to an average loss of 8.6 months of life expectancy in Europe [44]. Data also estimate that over 3 million life-years were lost in the EU ( 25 countries) in 2000 through exposure to particulate matter [44]. Estimates of loss in statistical life expectancy in the UK that can be attributed to anthropogenic PM2.5 sources were 6.9 months in 2000 and 4.9 months in 2010. Experts estimate that the "no further climate measures" scenario for 2020 will still lead to a loss of life expectancy of 4.5 months in the UK. Previous data on chronic exposure, combined with our findings on acute pollution fluctuations, suggest that caregivers should inform patients with bronchiectasis on the effects of air pollution on their disease. However, it remains to be established if certain interventions (such as mask protection during peak exposure) will impact disease morbidity. Our analysis might in part provide an answer to that question. An intervention that would decrease PM10 by $10 \mu \mathrm{g} \cdot \mathrm{m}^{-3}$ would potentially lead to a reduction in exacerbations of $11.2 \%$. This means that during the study period, a reduction in PM10 of $10 \mu \mathrm{g} \cdot \mathrm{m}^{-3}$ would possibly prevent 871 exacerbations, or 59 exacerbations per year. For $\mathrm{NO}_{2}$, a decrease of $10 \mu \mathrm{g} \cdot \mathrm{m}^{-3}$ would likely prevent 25 exacerbations each year. Further research should focus on unravelling whether certain patients have a particular sensitivity to air pollution. This could then lead to research testing interventions and patient education programmes to improve their health status.

In conclusion, acute air pollution fluctuations are associated with increased exacerbation risk in patients with bronchiectasis. There was no difference in risk between patients stratified according to BSI, deprivation index or the presence of chronic $P$. aeruginosa infection, but there was a greater effect during the spring and especially during the summer months. Air pollution seems to be an important factor in bronchiectasis and patients should be aware of its effects.

Acknowledgements: We acknowledge the support of the Health Informatics Centre (HIC), University of Dundee, the Farr Institute Scotland for managing and supplying the anonymised data and NHS Tayside, the original data source.

Author contribution: P.C. Goeminne wrote the article and contributed to the design of the study. B. Cox and T.S. Nawrot contributed to the design of the study, performed the statistical analysis and reviewed the paper. T.C. Fardon, S. Finch, M.R. Loebinger, P. Bedi and A.T. Hill contributed to the design and review of the manuscript. K. de Hoogh and J.D. Chalmers contributed to the design of the study, collected the data and wrote the article.

Conflict of interest: A.T. Hill reports personal fees for advisory board activities from Bayer, outside the submitted work. J.D. Chalmers reports grants from GlaxoSmithKline, grants and personal fees from Boehringer Ingelheim, Pfizer, AstraZeneca and Bayer Healthcare, and personal fees from Grifols and Chiesi, outside the submitted work. P.C. Goeminne reports personal fees for lecturing from AstraZeneca, personal fees for lecturing and support for attending conferences from Chiesi, Boehringer and Bayer, and support for attending conferences from Pfizer, outside the submitted work.

Support statement: Funding was provided by the British Lung Foundation through the GSK/British Lung Foundation Chair of Respiratory Research (J.D. Chalmers) and by the European Respiratory Society through the EMBARC (European Multicentre Audit and Research Collaboration) project. Funding information for this article has been deposited with the Crossref Funder Registry.

\section{References}

1 Chalmers JD, Goeminne PC, Aliberti S, et al. The Bronchiectasis Severity Index: an international derivation and validation study. Am J Respir Crit Care Med 2014; 189: 576-585.

2 Chalmers JD, Aliberti S, Filonenko A, et al. Characterisation of the "frequent exacerbator phenotype" in bronchiectasis. Am J Respir Crit Care Med 2018; 197: 1410-1420.

3 Polverino E, Goeminne PC, McDonnell MJ, et al. European Respiratory Society guidelines for the management of adult bronchiectasis. Eur Respir J 2017; 50: 1700629.

4 Chalmers JD, Haworth CS, Johnson C, et al. Management of bronchiectasis in Europe: data from the European Bronchiectasis Registry (EMBARC). Eur Respir J 2016; 48: Suppl. 60, OA273.

5 McCreanor J, Cullinan P, Nieuwenhuijsen MJ, et al. Respiratory effects of exposure to diesel traffic in persons with asthma. N Engl J Med 2007; 357: 2348-2358. 
6 Angrill J, Agustí C, De Celis R, et al. Bronchial inflammation and colonization in patients with clinically stable bronchiectasis. Am J Respir Crit Care Med 2001; 164: 1628-1632.

7 Goeminne PC, Kiciński M, Vermeulen F, et al. Impact of air pollution on cystic fibrosis pulmonary exacerbations a case-crossover analysis. Chest 2013; 143: 946-954.

8 Farhat SC, Almeida MB, Silva-Filho LV, et al. Ozone is associated with an increased risk of respiratory exacerbations in patients with cystic fibrosis. Chest 2013; 144: 1186-1192.

9 Jassal MS, Yu AM, Bhatia R, et al. Effect of residential proximity to major roadways on cystic fibrosis exacerbations. Int J Env Health Res 2013; 23: 119-131.

10 Goss CH, Newsom SA, Schildcrout JS, et al. Effect of ambient air pollution on pulmonary exacerbations and lung function in cystic fibrosis. Am J Respir Crit Care Med 2004; 169: 816-821.

11 Goeminne PC, Bijnens E, Nemery B, et al. Impact of traffic related air pollution indicators on non-cystic fibrosis bronchiectasis mortality: a cohort analysis. Respir Res 2014; 15: 108.

12 Short PM, Anderson WJ, Elder DH, et al. Impact of left ventricular hypertrophy on survival in chronic obstructive pulmonary disease. Lung 2015; 193: 487-495.

13 Short PM, Lipworth SI, Elder DH, et al. Effect of beta-blockers in treatment of chronic obstructive disease: a retrospective cohort study. BMJ 2011; 342: d2549.

14 Schembri S, Williamson PA, Short PM, et al. Cardiovascular events following Clarithromycin use in lower respiratory tract infections: analysis of two prospective cohort studies. BMJ 2013; 346: f1235.

15 Ye X, Wolff R, Yu W, et al. Ambient temperature and morbidity: a review of epidemiological evidence. Environ Health Perspect 2012; 120: 19-28.

16 Nawrot TS, Perez L, Kunzli N, et al. Public health importance of triggers of myocardial infarction: a comparative risk assessment. Lancet 2011; 377: 732-740.

17 Maclure M. The case-crossover design: a method for studying transient effects on the risk of acute events. Am J Epidemiol 1991; 133: 144-153.

18 Levy D, Lumley T, Sheppard L, et al. Referent selection in case-crossover analyses of acute health effects of air pollution. Epidemiology 2001; 12: 186-192.

19 Luo X, Sorock GS. Analysis of recurrent event data under the case-crossover design with applications to elderly falls. Stat Med 2008; 27: 2890-2901.

20 Gasparrini A. Modeling exposure-lag-response associations with distributed lag non-linear models. Stat Med 2014 33: 881-899.

21 Gasparrini A. Distributed lag linear and non-linear models in R: the package dlnm. J Stat Softw 2011; 43: 1-20.

22 Scottish Government. The Scottish Index of Multiple Deprivation. www.gov.scot/Topics/Statistics/SIMD/ Date last updated: June 06, 2018.

23 Dickie C. Guidance on the definition of SIMD quintiles. www.gov.scot/Resource/0043/00439496.pdf Date last updated: October, 2013.

24 Schwartz J. The distributed lag between air pollution and daily deaths. Epidemiology 2000; 11: 320-326.

25 Kunzli N, Schindler C. A call for reporting the relevant exposure term in air pollution case-crossover studies. J Epidemiol Community Health 2005; 59: 527-530.

26 Tramuto F, Cusimano R, Cerame G, et al. Urban air pollution and emergency room admissions for respiratory symptoms: a case-crossover study in Palermo, Italy. Environ Health 2011; 10: 31 .

27 Hetland RB, Cassee FR, Låg M, et al. Cytokine release from alveolar macrophages exposed to ambient particulate matter: heterogeneity in relation to size, city and season. Part Fibre Toxicol 2005; 2: 4

28 Sørensen $\mathrm{M}$, Loft $\mathrm{S}$, Andersen HV, et al. Personal exposure to $\mathrm{PM}_{2.5}$, black smoke and $\mathrm{NO}_{2}$ in Copenhagen: relationship to bedroom and outdoor concentrations covering seasonal variation. J Expo Anal Environ Epidemiol 2005; 15: 413-422.

29 Collaco JM, McGready J, Green DM, et al. Effect of temperature on cystic fibrosis lung disease and infections: a replicated cohort study. PLoS ONE 2011; 6: e27784.

30 Wong C, Jayaram L, Karalus N, et al. Azithromycin for prevention of exacerbations in non-cystic fibrosis bronchiectasis (EMBRACE): a randomised, double-blind, placebo-controlled trial. Lancet 2012; 380: 660-667.

31 Serisier DJ, Martin ML, McGuckin MA, et al. Effect of long-term, low-dose erythromycin on pulmonary exacerbations among patients with non-cystic fibrosis bronchiectasis: the BLESS randomized controlled trial, JAMA 2013; 309: 1260-1267.

32 Hill AT, Haworth CS, Aliberti S, et al. Pulmonary exacerbations in adults with bronchiectasis: a consensus definition for clinical research. Eur Respir J 2017; 49: 1700051.

33 Brook RD, Rajagopalan S, Pope CA3rd, et al. Particulate matter air pollution and cardiovascular disease: an update to the scientific statement from the American Heart Association. Circulation 2010; 121: 2331-2378.

34 Vanderstraeten $\mathrm{P}$, Forton $\mathrm{M}$, Brasseur $\mathrm{O}$, et al. Black carbon instead of particle mass concentration as an indicator for the traffic related particles in the Brussels capital region. JEP 2011; 2: 525-532.

35 Janssen NA, Lanki T, Hoek G, et al. Associations between ambient, personal, and indoor exposure to fine particulate matter constituents in Dutch and Finnish panels of cardiovascular patients. Occup Environ Med 2005; 62: 868-877.

36 Williams R, Suggs J, Zweidinger R, et al. The 1998 Baltimore Particulate Matter Epidemiology-Exposure Study: part 1. Comparison of ambient, residential outdoor, indoor and apartment particulate matter monitoring. J Expo Anal Environ Epidemiol. 2000; 10: 518-532.

37 Scheers H, Mwalili SM, Faes C, et al. Does air pollution trigger infant mortality in Western Europe? A case-crossover study. Environ Health Perspect 2011; 119: 1017-1022.

38 Roberts ME, Lowndes L, Milne DG, et al. Socioeconomic deprivation, readmissions, mortality and acute exacerbations of bronchiectasis. Intern Med J 2012; 42: e129-e136.

39 Mao B, Yang JW, Lu HW, et al. Asthma and bronchiectasis exacerbation. Eur Respir J 2016; 47: 1680-1686.

40 Guarneri M, Balmes JR. Outdoor air pollution and asthma. Lancet 2014; 383: 1581-1592.

41 Goeminne PC, Nawrot TS, Ruttens D, et al. Mortality in non-cystic fibrosis bronchiectasis: a prospective cohort analysis. Respir Med 2014; 108: 287-296.

42 Gatheral T, Kumar N, Sansom B, et al. COPD-related bronchiectasis; independent impact on disease course and outcomes. COPD 2014; 11: 605-614. 
43 Gan WQ, FitzGerald JM, Carlsten C, et al. Associations of ambient air pollution with chronic obstructive pulmonary disease hospitalization and mortality. Am J Respir Crit Care Med 2013; 187: 721-727.

44 World Health Organization. Health risks of particulate matter from long-range transboundary air pollution. Copenhagen, World Health Organization Regional Office for Europe, 2006. www.euro.who.int/__data/assets/pdf_ file/0006/78657/E88189.pdf Date last accessed: July 12, 2017. 